\title{
New ergodic convergence theorems for non-expansive mappings and $m$-accretive mappings
}

\author{
Li Wei ${ }^{1 *}$, Yuchen Ba² and Ravi P Agarwal ${ }^{3,4}$
}

\section{"Correspondence:}

diandianba@yahoo.com

'School of Mathematics and

Statistics, Hebei University of

Economics and Business,

Shijiazhuang, 050061, China

Full list of author information is

available at the end of the article

\begin{abstract}
Two new ergodic convergence theorems for approximating the common element of the set of zero points of an $m$-accretive mapping and the set of fixed points of an infinite family of non-expansive mappings in a real smooth and uniformly convex Banach space are obtained, which improves some of the previous work. The computational experiments to demonstrate the effectiveness of the proposed iterative algorithms in this paper are conducted.
\end{abstract}

MSC: $47 \mathrm{H} 05 ; 47 \mathrm{H} 09 ; 47 \mathrm{H} 10$

Keywords: $m$-accretive mapping; non-expansive mapping; contraction; retraction; ergodic convergence; smooth Banach space

\section{Introduction and preliminaries}

Let $E$ be a real Banach space with norm $\|\cdot\|$ and let $E^{*}$ denote the dual space of $E$. We use ' $\rightarrow$ ' and ' $\rightarrow$ ' (or ' $w$-lim') to denote strong and weak convergence either in $E$ or in $E^{*}$, respectively. We denote the value of $f \in E^{*}$ at $x \in E$ by $\langle x, f\rangle$.

A Banach space $E$ is said to be uniformly convex if, for each $\varepsilon \in(0,2]$, there exists $\delta>0$ such that

$$
\|x\|=\|y\|=1, \quad\|x-y\| \geq \varepsilon \quad \Rightarrow \quad\left\|\frac{x+y}{2}\right\| \leq 1-\delta .
$$

A Banach space $E$ is said to be smooth if

$$
\lim _{t \rightarrow 0} \frac{\|x+t y\|-\|x\|}{t}
$$

exists for each $x, y \in\{z \in E:\|z\|=1\}$.

The normalized duality mapping $J: E \rightarrow 2^{E^{*}}$ is defined by

$$
J x:=\left\{f \in E^{*}:\langle x, f\rangle=\|x\|^{2}=\|f\|^{2}\right\}, \quad x \in E .
$$

If $E$ is reduced to the Hilbert space $H$, then $J \equiv I$ is the identity mapping. If $E$ is smooth, then $J: E \rightarrow E^{*}$ is norm-to-norm continuous. The normalized duality mapping $J$ is said

(c) 2016 Wei et al. This article is distributed under the terms of the Creative Commons Attribution 4.0 International License (http://creativecommons.org/licenses/by/4.0/), which permits unrestricted use, distribution, and reproduction in any medium, provided you give appropriate credit to the original author(s) and the source, provide a link to the Creative Commons license, and indicate if changes were made. 
to be weakly sequentially continuous at zero if $\left\{x_{n}\right\}$ is a sequence in $E$ which converges weakly to 0 ; it follows that $\left\{J x_{n}\right\}$ converges in weak* to 0 .

For a mapping $T: D(T) \sqsubseteq E \rightarrow E$, we use $\operatorname{Fix}(T)$ to denote the fixed point set of it; that is, $\operatorname{Fix}(T):=\{x \in D(T): T x=x\}$.

Let $T: D(T) \sqsubseteq E \rightarrow E$ be a mapping. Then $T$ is said to be:

(1) non-expansive if

$$
\|T x-T y\| \leq\|x-y\| \quad \text { for } \forall x, y \in D(T) ;
$$

(2) contraction if there exists $0<k<1$ such that

$$
\|T x-T y\| \leq k\|x-y\| \quad \text { for } \forall x, y \in D(T) ;
$$

(3) accretive if, for all $x, y \in D(T)$, there exists $j(x-y) \in J(x-y)$ such that

$$
\langle T x-T y, j(x-y)\rangle \geq 0
$$

(4) $m$-accretive if $T$ is accretive and $R(I+\lambda T)=E$ for $\forall \lambda>0$;

(5) strongly positive (see [1]) if $D(T)=E$ where $E$ is a real smooth Banach space and there exists $\bar{\gamma}>0$ such that

$$
\langle T x, J x\rangle \geq \bar{\gamma}\|x\|^{2} \quad \text { for } \forall x \in E
$$

in this case,

$$
\|a I-b T\|=\sup _{\|x\| \leq 1}|\langle(a I-b T) x, J(x)\rangle|
$$

where $I$ is the identity mapping and $a \in[0,1], b \in[-1,1]$;

(6) demiclosed at $p$ if whenever $\left\{x_{n}\right\}$ is a sequence in $D(T)$ such that $x_{n} \rightarrow x \in D(T)$ and $T x_{n} \rightarrow p$ then $T x=p$.

For the accretive mapping $A$, we use $A^{-1} 0$ to denote the set of zero points of it; that is, $A^{-1} 0:=\{x \in D(A): A x=0\}$. If $A$ is accretive, then we can define, for each $r>0$, a nonexpansive single-valued mapping $J_{r}^{A}: R(I+r A) \rightarrow D(A)$ by $J_{r}^{A}:=(I+r A)^{-1}$, which is called the resolvent of $A$ [2]. It is well known that $J_{r}^{A}$ is non-expansive and $A^{-1} 0=\operatorname{Fix}\left(J_{r}^{A}\right)$.

Let $C$ be a nonempty, closed, and convex subset of $E$ and $Q$ be a mapping of $E$ onto $C$. Then $Q$ is said to be sunny [3] if $Q(Q(x)+t(x-Q(x)))=Q(x)$, for all $x \in E$ and $t \geq 0$.

A mapping $Q$ of $E$ into $E$ is said to be a retraction [3] if $Q^{2}=Q$. If a mapping $Q$ is a retraction, then $Q(z)=z$ for every $z \in R(Q)$, where $R(Q)$ is the range of $Q$.

A subset $C$ of $E$ is said to be a sunny non-expansive retract of $E$ [3] if there exists a sunny non-expansive retraction of $E$ onto $C$ and it is called a non-expansive retract of $E$ if there exists a non-expansive retraction of $E$ onto $C$.

The first mean ergodic theorem for non-expansive mappings was proved by Baillon in Hilbert space [4]. That is, for each $x \in C$, the Cesaro means

$$
S_{n}(x):=\frac{1}{n} \sum_{k=0}^{n-1} T^{k} x, \quad n \geq 1,
$$

converge weakly to a fixed point of a non-expansive mapping $T$. 
The implicit midpoint rule (IMR) for non-expansive mappings in a Hilbert space was introduced by [5]. This rule generates a sequence $\left\{x_{n}\right\}$ via the semi-implicit procedure:

$$
x_{n+1}=\left(1-t_{n}\right) x_{n}+t_{n} T\left(\frac{x_{n+1}+x_{n}}{2}\right), \quad n \geq 0 .
$$

$\left\{x_{n}\right\}$ is proved to be weakly convergent to a fixed point of the non-expansive mapping $T$.

The ergodic convergence of the sequence $\left\{x_{n}\right\}$ generated by (1.2) is considered in a Hilbert space in [6]. That is, the convergence of the means

$$
z_{n}:=\frac{1}{\sum_{k=1}^{n} a_{k}} \sum_{k=1}^{n} a_{k} x_{k}, \quad n=1,2, \ldots
$$

where $\left\{x_{k}\right\}$ satisfies (1.2), to a fixed point of the non-expansive mapping $T$ is obtained.

In a Hilbert space, Marino et al. presented the following iterative algorithm in [7]:

$$
x_{0} \in C, \quad x_{n+1}=\alpha_{n} \gamma f\left(x_{n}\right)+\left(I-\alpha_{n} A\right) T x_{n}, \quad n \geq 0,
$$

where $f$ is a contraction, $A$ is a strongly positive linear bounded operator, and $T$ is nonexpansive. If $\operatorname{Fix}(T) \neq \emptyset$, they proved that $\left\{x_{n}\right\}$ converges strongly to $p \in \operatorname{Fix}(T)$, which solves the variational inequality $\langle(\gamma f-A) p, z-p\rangle \leq 0$, for $\forall z \in \operatorname{Fix}(T)$ under some conditions.

Motivated by the previous work, we shall present two iterative algorithms and the ergodic convergence theorems are obtained. The connection between the strongly convergent point and the solution of one kind variational inequalities is being set up. Some examples are exemplified to illustrate the effectiveness of the proposed algorithms. The computational experiments are conducted and the codes are written in Visual Basic Six.

We need the following preliminaries.

Lemma 1.1 (see [8]) Let E be a Banach space and $C$ be a nonempty closed and convex subset of E. Let $f: C \rightarrow C$ be a contraction. Then $f$ has a unique fixed point $u \in C$.

Lemma 1.2 (see [9]) Let E be a real uniformly convex Banach space, $C$ be a nonempty, closed, and convex subset of $E$ and $T: C \rightarrow E$ be a non-expansive mapping such that $\operatorname{Fix}(T) \neq \emptyset$, then $I-T$ is demiclosed at zero.

Lemma 1.3 (see [10]) In a real Banach space E, the following inequality holds:

$$
\|x+y\|^{2} \leq\|x\|^{2}+2\langle y, j(x+y)\rangle, \quad \forall x, y \in E,
$$

where $j(x+y) \in J(x+y)$.

Lemma 1.4 (see [11]) Let $\left\{a_{n}\right\}$ and $\left\{c_{n}\right\}$ be two sequences of nonnegative real numbers satisfying

$$
a_{n+1} \leq\left(1-t_{n}\right) a_{n}+b_{n}+c_{n}, \quad \forall n \geq 0,
$$


where $\left\{t_{n}\right\} \subset(0,1)$ and $\left\{b_{n}\right\}$ is a number sequence. Assume that $\sum_{n=0}^{\infty} t_{n}=+\infty$, $\limsup _{n \rightarrow \infty} \frac{b_{n}}{t_{n}} \leq 0$, and $\sum_{n=0}^{\infty} c_{n}<+\infty$. Then $\lim _{n \rightarrow \infty} a_{n}=0$.

Lemma 1.5 (see [12]) Let $E$ be a Banach space and let $A$ be an m-accretive mapping. For $\lambda>0, \mu>0$, and $x \in E$, one has

$$
J_{\lambda}^{A} x=J_{\mu}^{A}\left(\frac{\mu}{\lambda} x+\left(1-\frac{\mu}{\lambda}\right) J_{\lambda}^{A} x\right)
$$

where $J_{\lambda}^{A}=(I+\lambda A)^{-1}$ and $J_{\mu}^{A}=(I+\mu A)^{-1}$.

Lemma 1.6 (see [13]) Assume $T$ is a strongly positive bounded operator with coefficient $\bar{\gamma}>0$ on a real smooth Banach space $E$ and $0<\rho \leq\|T\|^{-1}$. Then $\|I-\rho T\| \leq 1-\rho \bar{\gamma}$.

Lemma 1.7 (see [14]) Let E be a real smooth and uniformly convex Banach space and C be a nonempty, closed, and convex sunny non-expansive retract of $E$, and let $Q_{C}$ be the sunny non-expansive retraction of $E$ onto $C$. Let $f: E \rightarrow E$ be a fixed contractive mapping with coefficient $k \in(0,1), T: E \rightarrow E$ be a strongly positive linear bounded operator with coefficient $\bar{\gamma}$ and $U: C \rightarrow C$ be a non-expansive mapping. Suppose that the duality mapping $J: E \rightarrow E^{*}$ is weakly sequentially continuous at zero, $0<\eta<\frac{\bar{\gamma}}{2 k}$ and $\operatorname{Fix}(U) \neq \emptyset$. If for each $t \in(0,1)$, define $T_{t}: E \rightarrow E$ by

$$
T_{t} x:=\operatorname{t\eta f}(x)+(I-t T) U Q_{C} x,
$$

then $T_{t}$ has a fixed point $x_{t}$, for each $0<t \leq\|T\|^{-1}$, which is convergent strongly to the fixed point of $U$, as $t \rightarrow 0$. That is, $\lim _{t \rightarrow 0} x_{t}=p_{0} \in \operatorname{Fix}(U)$. Moreover, $p_{0}$ satisfies the following variational inequality: for $\forall z \in \operatorname{Fix}(U)$,

$$
\left\langle(T-\eta f) p_{0}, J\left(p_{0}-z\right)\right\rangle \leq 0
$$

Lemma 1.8 (see [15]) Let $E$ be a real strictly convex Banach space and let $C$ be a nonempty closed and convex subset of $E$. Let $T_{m}: C \rightarrow C$ be a non-expansive mapping for each $m \geq 1$. Let $\left\{a_{m}\right\}$ be a real number sequence in $(0,1)$ such that $\sum_{m=1}^{\infty} a_{m}=1$. Suppose that $\bigcap_{m=1}^{\infty} \operatorname{Fix}\left(T_{m}\right) \neq \emptyset$. Then the mapping $\sum_{m=1}^{\infty} a_{m} T_{m}$ is non-expansive with $\operatorname{Fix}\left(\sum_{m=1}^{\infty} a_{m} T_{m}\right)=\bigcap_{m=1}^{\infty} \operatorname{Fix}\left(T_{m}\right)$.

\section{Strong convergence theorems}

Lemma 2.1 Suppose $A: D(A) \subset E \rightarrow E$ is an m-accretive mapping, where $E$ is a Banach space, and $\left\{r_{n}\right\}_{n=0}^{\infty} \subset(0,+\infty)$ is any real number sequence. Then, for $n \geq 0, \forall x, y \in E$, if $r_{n} \leq r_{n+1}$,

$$
\left\|J_{r_{n+1}}^{A} x-J_{r_{n}}^{A} y\right\| \leq\|x-y\|+\frac{r_{n+1}-r_{n}}{r_{n+1}}\left\|J_{r_{n+1}}^{A} x-y\right\| ;
$$

if $r_{n+1} \leq r_{n}$,

$$
\left\|J_{r_{n+1}}^{A} x-J_{r_{n}}^{A} y\right\| \leq\|x-y\|+\frac{r_{n}-r_{n+1}}{r_{n}}\left\|x-J_{r_{n}}^{A} y\right\| .
$$


Proof In fact, if $r_{n} \leq r_{n+1}$, then, using Lemma 1.5, we have

$$
\begin{aligned}
\left\|J_{r_{n+1}}^{A} x-J_{r_{n}}^{A} y\right\| & =\left\|J_{r_{n}}^{A}\left[\frac{r_{n}}{r_{n+1}} x+\left(1-\frac{r_{n}}{r_{n+1}}\right) J_{r_{n+1}}^{A} x\right]-J_{r_{n}}^{A} y\right\| \\
& \leq \frac{r_{n}}{r_{n+1}}\|x-y\|+\left(1-\frac{r_{n}}{r_{n+1}}\right)\left\|J_{r_{n+1}}^{A} x-y\right\| \\
& \leq\|x-y\|+\frac{r_{n+1}-r_{n}}{r_{n+1}}\left\|J_{r_{n+1}}^{A} x-y\right\|,
\end{aligned}
$$

which implies that (2.1) is true.

If $r_{n+1} \leq r_{n}$, then imitating the proof of (2.1), we have (2.2).

This completes the proof.

\subsection{Ergodic convergence of the first iterative algorithm}

Theorem 2.2 Let $E$ be a real smooth and uniformly convex Banach space. Let $C$ be a nonempty, closed, and convex sunny non-expansive retract of $E$, and $Q_{C}$ be the sunny nonexpansive retraction of $E$ onto $C$. Let $f: C \rightarrow C$ be a contraction with contractive constant $k \in(0,1), T: C \rightarrow C$ be a strongly positive linear bounded operator with coefficient $\bar{\gamma}$. Let $A: C \rightarrow E$ be an m-accretive mapping, and $S_{i}: C \rightarrow C$ be non-expansive mappings, for $i=1,2, \ldots$. Let $\Omega:=\bigcap_{i=1}^{\infty} \operatorname{Fix}\left(S_{i}\right) \cap A^{-1} 0 \neq \emptyset$. Suppose the duality mapping $J: E \rightarrow E^{*}$ is weakly sequentially continuous at zero, and $0<\eta<\frac{\bar{\gamma}}{2 k}$. Suppose $\left\{\alpha_{n, i}\right\},\left\{\delta_{n}\right\},\left\{\beta_{n}\right\},\left\{b_{n, i}\right\}$, $\left\{\gamma_{n}\right\} \subset(0,1)$ and $\left\{r_{n}\right\} \subset(0,+\infty)$, where $n \geq 0$ and $i=1,2, \ldots$. Let $\left\{x_{n}\right\}$ be generated by the following iterative algorithm:

$$
\left\{\begin{array}{l}
x_{0} \in C, \\
y_{n}=\beta_{n} x_{n}+\left(1-\beta_{n}\right) \sum_{i=1}^{\infty} b_{n, i}\left[\left(1-\alpha_{n, i}\right) J_{r_{n}}^{A}+\alpha_{n, i} S_{i}\right] Q_{C} x_{n}, \\
u_{n}=\left(1-\delta_{n}\right) y_{n}+\delta_{n} J_{r_{n}}^{A}\left(\frac{u_{n}+y_{n}}{2}\right), \\
x_{n+1}=\gamma_{n} \eta f\left(x_{n}\right)+\left(I-\gamma_{n} T\right) u_{n}, \quad n \geq 0,
\end{array}\right.
$$

where $J_{r_{n}}^{A}=\left(I+r_{n} A\right)^{-1}$, for $n \geq 0$. Further suppose that the following conditions are satisfied:

(i) $\sum_{n=0}^{\infty} \sum_{i=1}^{\infty}\left|\alpha_{n+1, i}-\alpha_{n, i}\right|<+\infty$;

(ii) $\sum_{n=0}^{\infty} \gamma_{n}=\infty, \gamma_{n} \rightarrow 0$, as $n \rightarrow \infty$ and $\sum_{n=1}^{\infty}\left|\gamma_{n}-\gamma_{n-1}\right|<+\infty$;

(iii) $\sum_{n=0}^{\infty}\left|r_{n+1}-r_{n}\right|<+\infty$ and $0<\varepsilon \leq r_{n}$, for $n \geq 0$;

(iv) $\sum_{n=1}^{\infty}\left|\delta_{n}-\delta_{n-1}\right|<+\infty, \sum_{n=1}^{\infty}\left|\beta_{n}-\beta_{n-1}\right|<+\infty, \beta_{n} \rightarrow 0, \delta_{n} \rightarrow 0$, as $n \rightarrow \infty$;

(v) $\sum_{n=0}^{\infty} \sum_{i=1}^{\infty}\left|b_{n+1, i}-b_{n, i}\right|<+\infty$ and $\sum_{i=1}^{\infty} b_{n, i}=1$ for $n \geq 0$.

Then the three sequences $\left\{y_{n}\right\},\left\{u_{n}\right\}$, and $\left\{x_{n}\right\}$ converge strongly to the unique element $q_{0} \in \Omega$ which satisfies the following variational inequality: for $\forall y \in \Omega$,

$$
\left\langle(T-\eta f) q_{0}, J\left(q_{0}-y\right)\right\rangle \leq 0
$$

Moreover, the ergodic convergence is obtained in the sense that

$$
z_{n}:=\frac{1}{\sum_{k=1}^{n} a_{k}} \sum_{k=1}^{n} a_{k} x_{k}, \quad n \geq 1,
$$

converges strongly to the above $q_{0}$, under the assumption that $\left\{a_{n}\right\}$ is a sequence of positive numbers such that $\sum_{k=1}^{n} a_{k} \rightarrow \infty$, as $n \rightarrow \infty$. 
To prove Theorem 2.2, we need the following lemmas.

Lemma 2.3 In Theorem 2.2, set $W_{n, i}=\left(1-\alpha_{n, i}\right) J_{r_{n}}^{A}+\alpha_{n, i} S_{i}$. Then $W_{n, i}: C \rightarrow C$ is nonexpansive for $n \geq 0$ and $i=1,2, \ldots$. Moreover, $\Omega=\bigcap_{i=1}^{\infty} \operatorname{Fix}\left(W_{n, i}\right)$.

Proof It can be easily obtained that $W_{n, i}: C \rightarrow C$ is non-expansive since both $J_{r_{n}}^{A}$ and $S_{i}$ are non-expansive, for $n \geq 0$ and $i=1,2, \ldots$.

The fact that $\Omega \subset \bigcap_{i=1}^{\infty} \operatorname{Fix}\left(W_{n, i}\right)$ is trivial. We are left to show that $\bigcap_{i=1}^{\infty} \operatorname{Fix}\left(W_{n, i}\right) \subset \Omega$.

For $p \in \bigcap_{i=1}^{\infty} \operatorname{Fix}\left(W_{n, i}\right)$, then $p=\left(1-\alpha_{n, i}\right) J_{r_{n}}^{A} p+\alpha_{n, i} S_{i} p$. For $\forall q \in \Omega \subset \bigcap_{i=1}^{\infty} \operatorname{Fix}\left(W_{n, i}\right)$, we have

$$
\begin{aligned}
\|p-q\| & =\left\|\left(1-\alpha_{n, i}\right)\left(J_{r_{n}}^{A} p-J_{r_{n}}^{A} q\right)+\alpha_{n, i}\left(S_{i} p-S_{i} q\right)\right\| \\
& \leq\left(1-\alpha_{n, i}\right)\left\|J_{r_{n}}^{A} p-q\right\|+\alpha_{n, i}\left\|S_{i} p-q\right\| \\
& \leq\left(1-\alpha_{n, i}\right)\left\|J_{r_{n}}^{A} p-q\right\|+\alpha_{n, i}\|p-q\|
\end{aligned}
$$

which implies that $\left\|J_{r_{n}}^{A} p-q\right\|=\|p-q\|$. Similarly, $\|p-q\|=\left\|S_{i} p-q\right\|$. Since $E$ is uniformly convex, we have $p-q=J_{r_{n}}^{A} p-q=S_{i} p-q$, which implies that $p \in \bigcap_{i=1}^{\infty} \operatorname{Fix}\left(S_{i}\right) \cap A^{-1} 0$.

This completes the proof.

Lemma 2.4 In Theorem 2.2, $\left\{z_{n}\right\}$ is well-defined.

Proof In fact, it suffices to show that $\left\{u_{n}\right\}$ is well-defined.

For $t \in(0,1)$, define $U_{t}: C \rightarrow C$ by $U_{t} x:=(1-t) y+t U\left(\frac{x+y}{2}\right)$, where $U: C \rightarrow C$ is nonexpansive for $x, y \in C$. Then for $\forall x, y \in C$,

$$
\left\|U_{t} x-U_{t} z\right\| \leq t\left\|\frac{x+y}{2}-\frac{y+z}{2}\right\| \leq \frac{t}{2}\|x-z\|
$$

Thus $U_{t}$ is a contraction, which ensures from Lemma 1.1 that there exists $x_{t} \in C$ such that $U_{t} x_{t}=x_{t}$. That is, $x_{t}=(1-t) y+t U\left(\frac{y+x_{t}}{2}\right)$.

Note that $J_{r_{n}}^{A}$ is non-expansive, then $\left\{u_{n}\right\}$ is well-defined, and then $\left\{x_{n}\right\}$ and $\left\{z_{n}\right\}$ are all well-defined.

This completes the proof.

Lemma 2.5 The variational inequality (2.3) in Theorem 2.2 has a unique solution in $\Omega$.

Proof Using Lemmas 1.7, 1.8 and 2.3, we know that there exists $v_{t}$ such that $v_{t}=\operatorname{t\eta f}\left(v_{t}\right)+$ $(I-t T) \sum_{i=1}^{\infty} b_{n, i} W_{n, i} Q_{C} v_{t}$, for $t \in(0,1)$, where $W_{n, i}$ is the same as that in Lemma 2.3, for $i=1,2, \ldots$. Moreover, $v_{t} \rightarrow q_{0} \in \Omega$, as $t \rightarrow 0$, which is the unique solution of the variational inequality (2.3).

This completes the proof.

Proof of Theorem 2.2 Step 1. $\left\{u_{n}\right\},\left\{y_{n}\right\}$, and $\left\{x_{n}\right\}$ are all bounded.

For $\forall p \in \Omega$, noticing Lemma 2.3, we see that for $n \geq 0$,

$$
\left\|y_{n}-p\right\| \leq\left\|x_{n}-p\right\| \text {. }
$$


Wei et al. Journal of Inequalities and Applications （2016） 2016:22

Page 7 of 20

Also,

$$
\left\|u_{n}-p\right\| \leq\left(1-\delta_{n}\right)\left\|y_{n}-p\right\|+\delta_{n}\left\|\frac{y_{n}+u_{n}}{2}-p\right\| \leq\left(1-\frac{\delta_{n}}{2}\right)\left\|y_{n}-p\right\|+\frac{\delta_{n}}{2}\left\|u_{n}-p\right\|,
$$

which implies that

$$
\left\|u_{n}-p\right\| \leq\left\|y_{n}-p\right\| \leq\left\|x_{n}-p\right\|
$$

Using Lemma 1.6 and (2.5), we have, for $n \geq 0$,

$$
\begin{aligned}
\left\|x_{n+1}-p\right\| & \leq \gamma_{n} \eta k\left\|x_{n}-p\right\|+\gamma_{n}\|\eta f(p)-T p\|+\left(1-\gamma_{n} \bar{\gamma}\right)\left\|u_{n}-p\right\| \\
& \leq\left[1-\gamma_{n}(\bar{\gamma}-k \eta)\right]\left\|x_{n}-p\right\|+\gamma_{n}\|\eta f(p)-T p\| .
\end{aligned}
$$

By using the inductive method, we can easily get the following result from (2.6):

$$
\left\|x_{n+1}-p\right\| \leq \max \left\{\left\|x_{0}-p\right\|, \frac{\|\eta f(p)-T p\|}{\bar{\gamma}-k \eta}\right\} \text {. }
$$

Therefore, $\left\{x_{n}\right\}$ is bounded. Then both $\left\{y_{n}\right\}$ and $\left\{u_{n}\right\}$ are bounded in view of (2.5).

Moreover, we can easily know that $\left\{J_{r_{n}}^{A} Q_{C} x_{n}\right\},\left\{S_{i} Q_{C} x_{n}\right\},\left\{J_{r_{n}}^{A}\left(\frac{u_{n}+y_{n}}{2}\right)\right\},\left\{Q_{C} x_{n}\right\},\left\{f\left(x_{n}\right)\right\}$, and $\left\{W_{n, i} Q_{C} x_{n}\right\}$ are all bounded, for $n \geq 0$ and $i=1,2, \ldots$.

Set

$$
\begin{aligned}
M^{\prime}= & \sup \left\{\left\|u_{n}\right\|,\left\|x_{n}\right\|,\left\|y_{n}\right\|,\left\|S_{i} Q_{C} x_{n}\right\|,\left\|J_{r_{n}}^{A}\left(\frac{u_{n}+y_{n}}{2}\right)\right\|,\left\|J_{r_{n}}^{A} Q_{C} x_{n}\right\|,\left\|Q_{C} x_{n}\right\|,\left\|f\left(x_{n}\right)\right\|,\right. \\
& \left.\left\|W_{n, i} Q_{C} x_{n}\right\|: n \geq 0, i \geq 1\right\} .
\end{aligned}
$$

Then $M^{\prime}$ is a positive constant.

Step 2. $\lim _{n \rightarrow \infty}\left\|x_{n+1}-x_{n}\right\|=0$.

In fact, using Lemma 2.1, we have, for $n \geq 0$,

$$
\begin{aligned}
& \left\|J_{r_{n+1}}^{A}\left(\frac{u_{n+1}+y_{n+1}}{2}\right)-J_{r_{n}}^{A}\left(\frac{u_{n}+y_{n}}{2}\right)\right\| \\
& \quad \leq \frac{\left\|u_{n+1}-u_{n}\right\|}{2}+\frac{\left\|y_{n+1}-y_{n}\right\|}{2}+2 \frac{\left|r_{n}-r_{n+1}\right|}{\varepsilon} M^{\prime} .
\end{aligned}
$$

In view of (2.7) and Lemma 2.1, we have, for $n \geq 0$,

$$
\begin{aligned}
\left\|u_{n+1}-u_{n}\right\| & \\
\leq & \left(1-\delta_{n+1}\right)\left\|y_{n+1}-y_{n}\right\|+\left|\delta_{n+1}-\delta_{n}\right|\left\|y_{n}\right\| \\
& +\delta_{n+1}\left\|J_{r_{n+1}}^{A}\left(\frac{u_{n+1}+y_{n+1}}{2}\right)-J_{r_{n}}^{A}\left(\frac{u_{n}+y_{n}}{2}\right)\right\|+\left|\delta_{n+1}-\delta_{n}\right|\left\|J_{r_{n}}^{A}\left(\frac{u_{n}+y_{n}}{2}\right)\right\| \\
\leq & \left(1-\delta_{n+1}\right)\left\|y_{n+1}-y_{n}\right\|+2\left|\delta_{n+1}-\delta_{n}\right| M^{\prime} \\
& +\frac{\delta_{n+1}}{2}\left(\left\|u_{n+1}-u_{n}\right\|+\left\|y_{n+1}-y_{n}\right\|\right)+\frac{2}{\varepsilon} \delta_{n+1}\left|r_{n}-r_{n+1}\right| M^{\prime},
\end{aligned}
$$


which implies that

$$
\begin{aligned}
\left\|u_{n+1}-u_{n}\right\| & \leq\left\|y_{n+1}-y_{n}\right\|+\frac{4\left|\delta_{n+1}-\delta_{n}\right| M^{\prime}}{2-\delta_{n+1}}+\frac{4 \delta_{n+1}\left|r_{n}-r_{n+1}\right| M^{\prime}}{\left(2-\delta_{n+1}\right) \varepsilon} \\
& \leq\left\|y_{n+1}-y_{n}\right\|+4\left|\delta_{n+1}-\delta_{n}\right| M^{\prime}+\frac{4 M^{\prime}}{\varepsilon}\left|r_{n+1}-r_{n}\right| .
\end{aligned}
$$

Now, in view of Lemma 2.1, computing the following:

$$
\begin{aligned}
&\left\|W_{n+1, i} Q_{C} x_{n+1}-W_{n, i} Q_{C} x_{n}\right\| \\
&=\left\|\left[\left(1-\alpha_{n+1, i}\right) J_{r_{n+1}}^{A}+\alpha_{n+1, i} S_{i}\right] Q_{C} x_{n+1}-\left[\left(1-\alpha_{n, i}\right) J_{r_{n}}^{A}+\alpha_{n, i} S_{i}\right] Q_{C} x_{n}\right\| \\
& \leq\left(1-\alpha_{n+1, i}\right)\left\|J_{r_{n+1}}^{A} Q_{C} x_{n+1}-J_{r_{n}}^{A} Q_{C} x_{n}\right\| \\
&+\alpha_{n+1, i}\left\|S_{i} Q_{C} x_{n+1}-S_{i} Q_{C} x_{n}\right\| \\
&+\left|\alpha_{n+1, i}-\alpha_{n, i}\right|\left(\left\|S_{i} Q_{C} x_{n}\right\|+\left\|J_{r_{n}}^{A} Q_{C} x_{n}\right\|\right) \\
& \leq\left(1-\alpha_{n+1, i}\right)\left\|x_{n+1}-x_{n}\right\|+2 M^{\prime} \frac{\left|r_{n+1}-r_{n}\right|}{\varepsilon} \\
&+\alpha_{n+1, i}\left\|x_{n+1}-x_{n}\right\|+2 M^{\prime}\left|\alpha_{n+1, i}-\alpha_{n, i}\right| \\
&=\left\|x_{n+1}-x_{n}\right\|+2 M^{\prime} \frac{\left|r_{n+1}-r_{n}\right|}{\varepsilon}+2 M^{\prime}\left|\alpha_{n+1, i}-\alpha_{n, i}\right| .
\end{aligned}
$$

Using (2.9), we have, for $n \geq 0$,

$$
\begin{aligned}
& \left\|\sum_{i=1}^{\infty} b_{n+1, i} W_{n+1, i} Q_{C} x_{n+1}-\sum_{i=1}^{\infty} b_{n, i} W_{n, i} Q_{C} x_{n}\right\| \\
& \leq\left\|\sum_{i=1}^{\infty} b_{n+1, i} W_{n+1, i} Q_{C} x_{n+1}-\sum_{i=1}^{\infty} b_{n+1, i} W_{n, i} Q_{C} x_{n}\right\| \\
& \quad+\left\|\sum_{i=1}^{\infty} b_{n+1, i} W_{n, i} Q_{C} x_{n}-\sum_{i=1}^{\infty} b_{n, i} W_{n, i} Q_{C} x_{n}\right\| \\
& \leq \sum_{i=1}^{\infty} b_{n+1, i}\left\|W_{n+1, i} Q_{C} x_{n+1}-W_{n, i} Q_{C} x_{n}\right\| \\
& \quad+\sum_{i=1}^{\infty}\left|b_{n+1, i}-b_{n, i}\right|\left\|W_{n, i} Q_{C} x_{n}\right\| \\
& \leq \\
& \quad\left\|x_{n+1}-x_{n}\right\|+2 M^{\prime} \frac{\left|r_{n+1}-r_{n}\right|}{\varepsilon}+2 M^{\prime} \sum_{i=1}^{\infty}\left|\alpha_{n+1, i}-\alpha_{n, i}\right| \\
& \quad+M^{\prime} \sum_{i=1}^{\infty}\left|b_{n+1, i}-b_{n, i}\right| .
\end{aligned}
$$

Using (2.10), we know that

$$
\begin{aligned}
& \left\|y_{n+1}-y_{n}\right\| \\
& \quad \leq \beta_{n+1}\left\|x_{n+1}-x_{n}\right\|+\left|\beta_{n+1}-\beta_{n}\right|\left\|x_{n}\right\|
\end{aligned}
$$




$$
\begin{aligned}
& +\left(1-\beta_{n+1}\right)\left\|\sum_{i=1}^{\infty} b_{n+1, i} W_{n+1, i} Q_{C} x_{n+1}-\sum_{i=1}^{\infty} b_{n, i} W_{n, i} Q_{C} x_{n}\right\| \\
& +\left|\beta_{n+1}-\beta_{n}\right| \sum_{i=1}^{\infty} b_{n, i}\left\|W_{n, i} Q_{C} x_{n}\right\| \\
& \leq\left\|x_{n+1}-x_{n}\right\|+2\left|\beta_{n+1}-\beta_{n}\right| M^{\prime}+2 M^{\prime} \frac{\left|r_{n+1}-r_{n}\right|}{\varepsilon} \\
& +2 M^{\prime} \sum_{i=1}^{\infty}\left|\alpha_{n+1, i}-\alpha_{n, i}\right|+M^{\prime} \sum_{i=1}^{\infty}\left|b_{n+1, i}-b_{n, i}\right| .
\end{aligned}
$$

Thus in view of (2.8) and (2.11), we have, for $n \geq 0$,

$$
\begin{aligned}
\| x_{n+2} & -x_{n+1} \| \\
\leq & \gamma_{n+1} \eta k\left\|x_{n+1}-x_{n}\right\|+(1+\eta)\left|\gamma_{n+1}-\gamma_{n}\right| M^{\prime} \\
& +\left(1-\gamma_{n+1} \bar{\gamma}\right)\left[\left\|x_{n}-x_{n+1}\right\|+2 M^{\prime}\left|\beta_{n+1}-\beta_{n}\right|+2 M^{\prime} \frac{\left|r_{n+1}-r_{n}\right|}{\varepsilon}\right. \\
& \left.+M^{\prime} \sum_{i=1}^{\infty}\left|b_{n+1, i}-b_{n, i}\right|+2 M^{\prime} \sum_{i=1}^{\infty}\left|\alpha_{n+1, i}-\alpha_{n, i}\right|+4 M^{\prime}\left|\delta_{n+1}-\delta_{n}\right|+\frac{4 M^{\prime}}{\varepsilon}\left|r_{n+1}-r_{n}\right|\right] \\
= & {\left[1-\gamma_{n+1}(\bar{\gamma}-\eta k)\right]\left\|x_{n+1}-x_{n}\right\|+(1+\eta) M^{\prime}\left|\gamma_{n+1}-\gamma_{n}\right| } \\
& +\left(1-\gamma_{n+1} \bar{\gamma}\right)\left[2 M^{\prime} \sum_{i=1}^{\infty}\left|\alpha_{n+1, i}-\alpha_{n, i}\right|+2 M^{\prime}\left|\beta_{n+1}-\beta_{n}\right|\right. \\
& \left.+4 M^{\prime}\left|\delta_{n+1}-\delta_{n}\right|+M^{\prime} \sum_{i=1}^{\infty}\left|b_{n+1, i}-b_{n, i}\right|+\frac{6 M^{\prime}}{\varepsilon}\left|r_{n+1}-r_{n}\right|\right]
\end{aligned}
$$

Using Lemma 1.4, we have from (2.12) $\lim _{n \rightarrow \infty}\left\|x_{n+1}-x_{n}\right\|=0$. Since $\gamma_{n} \rightarrow 0$, we have $x_{n+1}-u_{n}=\gamma_{n}\left(\eta f\left(x_{n}\right)-T u_{n}\right) \rightarrow 0$, as $n \rightarrow \infty$, which implies that $x_{n}-u_{n} \rightarrow 0$, as $n \rightarrow \infty$.

Since $\delta_{n} \rightarrow 0$, we have $u_{n}-y_{n}=\delta_{n}\left[J_{r_{n}}^{A}\left(\frac{u_{n}+y_{n}}{2}\right)-y_{n}\right] \rightarrow 0$, as $n \rightarrow \infty$. Therefore, $x_{n}-y_{n} \rightarrow$ 0 , as $n \rightarrow \infty$.

Then

$$
\begin{aligned}
& \left\|y_{n}-\sum_{i=1}^{\infty} b_{n, i} W_{n, i} Q_{C} y_{n}\right\| \\
& \leq\left\|y_{n}-\sum_{i=1}^{\infty} b_{n, i} W_{n, i} Q_{C} x_{n}\right\|+\left\|\sum_{i=1}^{\infty} b_{n, i} W_{n, i}\left(Q_{C} x_{n}-Q_{C} y_{n}\right)\right\| \\
& \leq \beta_{n}\left\|x_{n}-\sum_{i=1}^{\infty} b_{n, i} W_{n, i} Q_{C} x_{n}\right\|+\left\|x_{n}-y_{n}\right\| \rightarrow 0,
\end{aligned}
$$

since $\beta_{n} \rightarrow 0$. Moreover, $x_{n+1}-\sum_{i=1}^{\infty} b_{n, i} W_{n, i} Q_{C} y_{n} \rightarrow 0$, as $n \rightarrow \infty$.

Step 3. $\lim \sup _{n \rightarrow+\infty}\left\langle\eta f\left(q_{0}\right)-T q_{0}, J\left(x_{n+1}-q_{0}\right)\right\rangle \leq 0$.

From Lemma 2.5, we know that $v_{t}=t \eta f\left(v_{t}\right)+(I-t T) \sum_{i=1}^{\infty} b_{n, i} W_{n, i} Q_{C} v_{t}$ for $t \in(0,1)$. Moreover, $v_{t} \rightarrow q_{0} \in \Omega$, as $t \rightarrow 0$. $q_{0}$ is the unique solution of the variational inequality (2.3). 
Since $\left\|v_{t}\right\| \leq\left\|v_{t}-q_{0}\right\|+\left\|q_{0}\right\|$, we see that $\left\{v_{t}\right\}$ is bounded, as $t \rightarrow 0$. Using Lemma 1.3, we have

$$
\begin{aligned}
\| v_{t}- & y_{n} \|^{2} \\
= & \left\|v_{t}-\sum_{i=1}^{\infty} b_{n, i} W_{n, i} Q_{C} y_{n}+\sum_{i=1}^{\infty} b_{n, i} W_{n, i} Q_{C} y_{n}-y_{n}\right\|^{2} \\
\leq & \left\|v_{t}-\sum_{i=1}^{\infty} b_{n, i} W_{n, i} Q_{C} y_{n}\right\|^{2}+2\left\langle\sum_{i=1}^{\infty} b_{n, i} W_{n, i} Q_{C} y_{n}-y_{n}, J\left(v_{t}-y_{n}\right)\right\rangle \\
= & \left\|t \eta f\left(v_{t}\right)+(I-t T) \sum_{i=1}^{\infty} b_{n, i} W_{n, i} Q_{C} v_{t}-\sum_{i=1}^{\infty} b_{n, i} W_{n, i} Q_{C} y_{n}\right\|^{2} \\
& +2\left\langle\sum_{i=1}^{\infty} b_{n, i} W_{n, i} Q_{C} y_{n}-y_{n}, J\left(v_{t}-y_{n}\right)\right\rangle \\
\leq & \left\|\sum_{i=1}^{\infty} b_{n, i} W_{n, i} Q_{C} v_{t}-\sum_{i=1}^{\infty} b_{n, i} W_{n, i} Q_{C} y_{n}\right\|^{2} \\
& +2 t\left\langle\eta f\left(v_{t}\right)-T \sum_{i=1}^{\infty} b_{n, i} W_{n, i} Q_{C} v_{t}, J\left(v_{t}-\sum_{i=1}^{\infty} b_{n, i} W_{n, i} Q_{C} y_{n}\right)\right\rangle \\
& +2\left\langle\sum_{i=1}^{\infty} b_{n, i} W_{n, i} Q_{C} y_{n}-y_{n}, J\left(v_{t}-y_{n}\right)\right\rangle \\
\leq & \left\|v_{t}-y_{n}\right\|^{2}+2 t\left\langle\eta f\left(v_{t}\right)-T \sum_{i=1}^{\infty} b_{n, i} W_{n, i} Q_{C} v_{t}, J\left(v_{t}-\sum_{i=1}^{\infty} b_{n, i} W_{n, i} Q_{C} y_{n}\right)\right\rangle \\
& +2\left\|\sum_{i=1}^{\infty} b_{n, i} W_{n, i} Q_{C} y_{n}-y_{n}\right\|\left\|v_{t}-y_{n}\right\|,
\end{aligned}
$$

which implies that

$$
\begin{aligned}
t\langle T & \left.\sum_{i=1}^{\infty} b_{n, i} W_{n, i} Q_{C} v_{t}-\eta f\left(v_{t}\right), J\left(v_{t}-\sum_{i=1}^{\infty} b_{n, i} W_{n, i} Q_{C} y_{n}\right)\right\rangle \\
\leq & \left\|\sum_{i=1}^{\infty} b_{n, i} W_{n, i} Q_{C} y_{n}-y_{n}\right\|\left\|v_{t}-y_{n}\right\| .
\end{aligned}
$$

So, $\lim _{t \rightarrow 0} \lim \sup _{n \rightarrow+\infty}\left\langle T \sum_{i=1}^{\infty} b_{n, i} W_{n, i} Q_{C} v_{t}-\eta f\left(v_{t}\right), J\left(v_{t}-\sum_{i=1}^{\infty} b_{n, i} W_{n, i} Q_{C} y_{n}\right)\right\rangle \leq 0$ in view of Step 2.

Since $v_{t} \rightarrow q_{0}$, we have $\sum_{i=1}^{\infty} b_{n, i} W_{n, i} Q_{C} v_{t} \rightarrow \sum_{i=1}^{\infty} b_{n, i} W_{n, i} Q_{C} q_{0}=Q_{C} q_{0}=q_{0}$, as $t \rightarrow 0$. Noticing the fact that

$$
\begin{aligned}
& \left\langle T q_{0}-\eta f\left(q_{0}\right), J\left(q_{0}-\sum_{i=1}^{\infty} b_{n, i} W_{n, i} Q_{C} y_{n}\right)\right\rangle \\
& =\left\langle T q_{0}-\eta f\left(q_{0}\right), J\left(q_{0}-\sum_{i=1}^{\infty} b_{n, i} W_{n, i} Q_{C} y_{n}\right)-J\left(v_{t}-\sum_{i=1}^{\infty} b_{n, i} W_{n, i} Q_{C} y_{n}\right)\right\rangle
\end{aligned}
$$




$$
\begin{aligned}
& +\left\langle T q_{0}-\eta f\left(q_{0}\right), J\left(v_{t}-\sum_{i=1}^{\infty} b_{n, i} W_{n, i} Q_{C} y_{n}\right)\right\rangle \\
= & \left\langle T q_{0}-\eta f\left(q_{0}\right), J\left(q_{0}-\sum_{i=1}^{\infty} b_{n, i} W_{n, i} Q_{C} y_{n}\right)-J\left(v_{t}-\sum_{i=1}^{\infty} b_{n, i} W_{n, i} Q_{C} y_{n}\right)\right\rangle \\
& +\left\langle T q_{0}-\eta f\left(q_{0}\right)-T \sum_{i=1}^{\infty} b_{n, i} W_{n, i} Q_{C} v_{t}+\eta f\left(v_{t}\right), J\left(v_{t}-\sum_{i=1}^{\infty} b_{n, i} W_{n, i} Q_{C} y_{n}\right)\right\rangle \\
& +\left\langle T \sum_{i=1}^{\infty} b_{n, i} W_{n, i} Q_{C} v_{t}-\eta f\left(v_{t}\right), J\left(v_{t}-\sum_{i=1}^{\infty} b_{n, i} W_{n, i} Q_{C} y_{n}\right)\right\rangle,
\end{aligned}
$$

we have $\lim \sup _{n \rightarrow+\infty}\left\langle T q_{0}-\eta f\left(q_{0}\right), J\left(q_{0}-\sum_{i=1}^{\infty} b_{n, i} W_{n, i} Q_{C} y_{n}\right)\right\rangle \leq 0$.

Since $\left\langle T q_{0}-\eta f\left(q_{0}\right), J\left(q_{0}-x_{n+1}\right)\right\rangle=\left\langle T q_{0}-\eta f\left(q_{0}\right), J\left(q_{0}-x_{n+1}\right)-J\left(q_{0}-\sum_{i=1}^{\infty} b_{n, i} W_{n, i} Q_{C} y_{n}\right)\right\rangle+$ $\left\langle T q_{0}-\eta f\left(q_{0}\right), J\left(q_{0}-\sum_{i=1}^{\infty} b_{n, i} W_{n, i} Q_{C} y_{n}\right)\right\rangle$ and $x_{n+1}-\sum_{i=1}^{\infty} b_{n, i} W_{n, i} Q_{C} y_{n} \rightarrow 0$ in view of Step 2, we have $\limsup _{n \rightarrow \infty}\left\langle\eta f\left(q_{0}\right)-T q_{0}, J\left(x_{n+1}-q_{0}\right)\right\rangle \leq 0$.

Step 4. $x_{n} \rightarrow q_{0}$, as $n \rightarrow+\infty$, where $q_{0} \in \Omega$ is the same as that in Step 3 .

Since

$$
\left\|u_{n}-q_{0}\right\| \leq\left(1-\delta_{n}\right)\left\|y_{n}-q_{0}\right\|+\delta_{n}\left\|\frac{y_{n}+u_{n}}{2}-q_{0}\right\|,
$$

we have

$$
\left\|u_{n}-q_{0}\right\| \leq\left\|y_{n}-q_{0}\right\| \leq \beta_{n}\left\|x_{n}-q_{0}\right\|+\left(1-\beta_{n}\right)\left\|x_{n}-q_{0}\right\|=\left\|x_{n}-q_{0}\right\| .
$$

Using Lemma 1.3, we have, for $n \geq 0$,

$$
\begin{aligned}
\| x_{n+1} & -q_{0} \|^{2} \\
= & \left\|\gamma_{n}\left(\eta f\left(x_{n}\right)-T q_{0}\right)+\left(I-\gamma_{n} T\right)\left(u_{n}-q_{0}\right)\right\|^{2} \\
\leq & \left(1-\gamma_{n} \bar{\gamma}\right)^{2}\left\|u_{n}-q_{0}\right\|^{2}+2 \gamma_{n}\left\langle\eta f\left(x_{n}\right)-T q_{0}, J\left(x_{n+1}-q_{0}\right)\right\rangle \\
\leq & \left(1-\gamma_{n} \bar{\gamma}\right)^{2}\left\|x_{n}-q_{0}\right\|^{2} \\
& +2 \gamma_{n} \eta\left\langle f\left(x_{n}\right)-f\left(q_{0}\right), J\left(x_{n+1}-q_{0}\right)-J\left(x_{n}-q_{0}\right)\right\rangle \\
& +2 \gamma_{n} \eta\left\langle f\left(x_{n}\right)-f\left(q_{0}\right), J\left(x_{n}-q_{0}\right)\right\rangle+2 \gamma_{n}\left\langle\eta f\left(q_{0}\right)-T q_{0}, J\left(x_{n+1}-q_{0}\right)\right\rangle \\
\leq & {\left[1-\gamma_{n}(\bar{\gamma}-2 \eta k)\right]\left\|x_{n}-q_{0}\right\|^{2} } \\
& +2 \gamma_{n}\left[\left\langle\eta f\left(q_{0}\right)-T q_{0}, J\left(x_{n+1}-q_{0}\right)\right\rangle+\eta\left\|x_{n}-q_{0}\right\|\left\|x_{n+1}-x_{n}\right\|\right] .
\end{aligned}
$$

Let $\delta_{n}^{(1)}=\gamma_{n}(\bar{\gamma}-2 \eta k), \delta_{n}^{(2)}=2 \gamma_{n}\left[\left\langle\eta f\left(q_{0}\right)-T q_{0}, J\left(x_{n+1}-q_{0}\right)\right\rangle+\eta\left\|x_{n}-q_{0}\right\|\left\|x_{n+1}-x_{n}\right\|\right]$. Then (2.13) can be simplified as $\left\|x_{n+1}-p_{0}\right\|^{2} \leq\left(1-\delta_{n}^{(1)}\right)\left\|x_{n}-q_{0}\right\|^{2}+\delta_{n}^{(2)}$.

Using the assumption (ii), the result of Steps 2 and 3 and Lemma 1.4, we know that $x_{n} \rightarrow q_{0}$, as $n \rightarrow+\infty$.

Combining with the result of Step 2, $y_{n} \rightarrow q_{0}$ and $u_{n} \rightarrow q_{0}$, as $n \rightarrow \infty$.

Step 5. $z_{n} \rightarrow q_{0}$, as $n \rightarrow \infty$.

Since $\sum_{k=1}^{n} a_{k} \rightarrow \infty$ and $\left\|x_{n}-q_{0}\right\| \rightarrow 0$, as $n \rightarrow \infty$, we have, for $\forall \varepsilon>0$, there exists $N^{*}$, such that, for all $n \geq N^{*}, \frac{1}{\sum_{k=1}^{n} a_{k}} \sum_{k=1}^{N^{*}} a_{k} M^{\prime \prime}<\frac{\varepsilon}{2}$ and $\left\|x_{n}-q_{0}\right\|<\frac{\varepsilon}{2}$, where $M^{\prime \prime}=\max \left\{\| x_{k}-\right.$ 
$\left.q_{0} \|: k=1,2, \ldots, N^{*}\right\}$. Then, for all $n>N^{*}$,

$$
\begin{aligned}
\left\|z_{n}-q_{0}\right\| & =\left\|\frac{1}{\sum_{k=1}^{n} a_{k}} \sum_{k=1}^{n} a_{k}\left(x_{k}-q_{0}\right)\right\| \\
& =\left\|\frac{1}{\sum_{k=1}^{n} a_{k}}\left[\sum_{k=1}^{N^{*}} a_{k}\left(x_{k}-q_{0}\right)+\sum_{k=N^{*}+1}^{n} a_{k}\left(x_{k}-q_{0}\right)\right]\right\| \\
& \leq \frac{1}{\sum_{k=1}^{n} a_{k}} \sum_{k=1}^{N^{*}} a_{k}\left\|x_{k}-q_{0}\right\|+\frac{1}{\sum_{k=1}^{n} a_{k}} \sum_{k=N^{*}+1}^{n} a_{k}\left\|x_{k}-q_{0}\right\| \\
& \leq \frac{1}{\sum_{k=1}^{n} a_{k}} \sum_{k=1}^{N^{*}} a_{k} M^{\prime \prime}+\frac{\sum_{k=N^{*}+1}^{n} a_{k}}{\sum_{k=1}^{n} a_{k}} \frac{\varepsilon}{2} \\
& <\frac{\varepsilon}{2}+\frac{\varepsilon}{2}=\varepsilon,
\end{aligned}
$$

which implies that $z_{n} \rightarrow q_{0}$, as $n \rightarrow \infty$.

This completes the proof.

Remark 2.6 The assumptions imposed on the real number sequences in Theorem 2.2 are reasonable if we take $\alpha_{n, i}=\frac{1}{(n+1) i^{2}}, \gamma_{n}=\delta_{n}=\beta_{n}=\frac{1}{1+n}, r_{n}=\varepsilon+\frac{1}{n+1}$, and $b_{n, i}=\frac{n+1}{(n+2)^{i}}$, for $n \geq 0$ and $i=1,2, \ldots$.

\subsection{Ergodic convergence of the second iterative algorithm}

Theorem 2.7 Let $E, \Omega, f, A, S_{i},\left\{r_{n}\right\},\left\{\delta_{n}\right\},\left\{b_{n, i}\right\}$, and $\left\{\alpha_{n, i}\right\}$ be the same as those in Theorem 2.2. Let $C$ be a nonempty, closed, and convex subset of $E$. Suppose $\Omega \neq \emptyset, 0<k<\frac{1}{2}$, the duality mapping $J: E \rightarrow E^{*}$ is weakly sequentially continuous at zero and $\left\{\zeta_{n}\right\} \subset(0,1)$. Let $\left\{x_{n}\right\}$ be generated by the following iterative algorithm:

$$
\left\{\begin{array}{l}
x_{0} \in C \\
u_{n}=\left(1-\delta_{n}\right) x_{n}+\delta_{n} J_{r_{n}}^{A}\left(\frac{u_{n}+x_{n}}{2}\right), \\
x_{n+1}=\left[\zeta_{n} f+\left(1-\zeta_{n}\right) I\right] \sum_{i=1}^{\infty} b_{n, i}\left[\left(1-\alpha_{n, i}\right) J_{r_{n}}^{A}+\alpha_{n, i} S_{i}\right] u_{n}, \quad n \geq 0 .
\end{array}\right.
$$

Further suppose that the following conditions are satisfied:

(vi) $\sum_{n=0}^{\infty} \zeta_{n}=\infty, \sum_{n=0}^{\infty}\left|\zeta_{n+1}-\zeta_{n}\right|<+\infty$, and $\zeta_{n} \rightarrow 0$, as $n \rightarrow \infty$.

Then both $\left\{u_{n}\right\}$ and $\left\{x_{n}\right\}$ converge strongly to the unique element $p_{0} \in \Omega$, which satisfies the following variational inequality: for $\forall y \in \Omega$,

$$
\left\langle p_{0}-f\left(p_{0}\right), J\left(p_{0}-y\right)\right\rangle \leq 0
$$

Moreover, the ergodic convergence is obtained in the sense that

$$
z_{n}:=\frac{1}{\sum_{k=1}^{n} a_{k}} \sum_{k=1}^{n} a_{k} x_{k}, \quad n \geq 1
$$

converges strongly to the above $p_{0}$ under the assumption that $\left\{a_{n}\right\}$ is a sequence of positive numbers such that $\sum_{k=1}^{n} a_{k} \rightarrow \infty$, as $n \rightarrow \infty$. 
Proof Using the same method as that in Theorem 2.2, we know that $\left\{u_{n}\right\}$ is well-defined. Let $V_{n}=\zeta_{n} f+\left(1-\zeta_{n}\right) I$.

Step 1. $V_{n}: C \rightarrow C$ is a contraction.

For $\forall x, y \in C$,

$$
\begin{aligned}
\left\|V_{n} x-V_{n} y\right\| & =\left\|\zeta_{n}(f(x)-f(y))+\left(1-\zeta_{n}\right)(x-y)\right\| \\
& \leq \zeta_{n} k\|x-y\|+\left(1-\zeta_{n}\right)\|x-y\|=\left[1-(1-k) \zeta_{n}\right]\|x-y\| .
\end{aligned}
$$

Step 2. $\left\{x_{n}\right\}$ is bounded.

Let $W_{n, i}$ be the same as that in Lemma 2.3 and Theorem 2.2, then for $p \in \Omega=$ $\bigcap_{i=1}^{\infty} \operatorname{Fix}\left(W_{n, i}\right)$, we can easily know that $\left\|u_{n}-p\right\| \leq\left\|x_{n}-p\right\|$. Using (2.15), we have

$$
\begin{aligned}
\left\|x_{n+1}-p\right\| & =\left\|V_{n} \sum_{i=1}^{\infty} b_{n, i} W_{n, i} u_{n}-V_{n} \sum_{i=1}^{\infty} b_{n, i} W_{n, i} p+V_{n} \sum_{i=1}^{\infty} b_{n, i} W_{n, i} p-p\right\| \\
& \leq\left[1-(1-k) \zeta_{n}\right]\left\|u_{n}-p\right\|+\zeta_{n}\|f(p)-p\| \\
& \leq\left[1-(1-k) \zeta_{n}\right]\left\|x_{n}-p\right\|+\zeta_{n}\|f(p)-p\| \\
& \leq \max \left\{\left\|x_{0}-p\right\|, \frac{\|f(p)-p\|}{1-k}\right\} .
\end{aligned}
$$

Therefore, $\left\{x_{n}\right\}$ is bounded. Thus $\left\{J_{r_{n}}^{A}\left(\frac{u_{n}+x_{n}}{2}\right)\right\},\left\{u_{n}\right\},\left\{W_{n, i} u_{n}\right\}$ and $\left\{f\left(\sum_{i=1}^{\infty} b_{n, i} W_{n, i} u_{n}\right)\right\}$ are all bounded for $n \geq 0$ and $i=1,2, \ldots$. Let

$$
\begin{aligned}
M^{\prime \prime \prime}= & \sup \left\{M^{\prime},\left\|x_{n}\right\|,\left\|u_{n}\right\|,\left\|J_{r_{n}}^{A}\left(\frac{u_{n}+x_{n}}{2}\right)\right\|,\left\|W_{n, i} u_{n}\right\|,\right. \\
& \left.\left\|f\left(\sum_{i=1}^{\infty} b_{n, i} W_{n, i} u_{n}\right)\right\|: n \geq 0, i=1,2, \ldots\right\},
\end{aligned}
$$

where $M^{\prime}$ is the same as that in Theorem 2.2, then $M^{\prime \prime \prime}$ is a positive constant.

Step 3. $\lim _{n \rightarrow \infty}\left\|x_{n+1}-x_{n}\right\|=0$.

In fact, using Lemma 2.1, we know that

$$
\begin{aligned}
& \left\|u_{n+1}-u_{n}\right\| \\
& \leq \quad\left(1-\delta_{n+1}\right)\left\|x_{n+1}-x_{n}\right\|+\left|\delta_{n+1}-\delta_{n}\right|\left\|x_{n}\right\|+\delta_{n+1}\left\|J_{r_{n+1}}^{A}\left(\frac{u_{n+1}+x_{n+1}}{2}\right)-J_{r_{n}}^{A}\left(\frac{u_{n}+x_{n}}{2}\right)\right\| \\
& \quad+\left|\delta_{n+1}-\delta_{n}\right|\left\|J_{r_{n}}^{A}\left(\frac{u_{n}+x_{n}}{2}\right)\right\| \\
& \leq \\
& \quad\left(1-\delta_{n+1}\right)\left\|x_{n+1}-x_{n}\right\|+2 M^{\prime \prime \prime}\left|\delta_{n+1}-\delta_{n}\right|+\delta_{n+1}\left(\frac{\left\|u_{n+1}-u_{n}\right\|}{2}+\frac{\left\|x_{n+1}-x_{n}\right\|}{2}\right) \\
& \quad+\frac{2 M^{\prime \prime \prime}}{\varepsilon}\left|r_{n+1}-r_{n}\right|
\end{aligned}
$$

which implies that $\left\|u_{n+1}-u_{n}\right\| \leq\left\|x_{n+1}-x_{n}\right\|+4\left|\delta_{n+1}-\delta_{n}\right| M^{\prime \prime \prime}+4 M^{\prime \prime \prime} \frac{\left|r_{n+1}-r_{n}\right|}{\varepsilon}$. 
Thus using (2.15) and noticing the result of (2.9), we have, for $n \geq 0$,

$$
\begin{aligned}
& \left\|x_{n+2}-x_{n+1}\right\| \\
& =\left\|V_{n+1} \sum_{i=1}^{\infty} b_{n+1, i} W_{n+1, i} u_{n+1}-V_{n} \sum_{i=1}^{\infty} b_{n, i} W_{n, i} u_{n}\right\| \\
& \leq V_{n+1} \sum_{i=1}^{\infty} b_{n+1, i} W_{n+1, i} u_{n+1}-V_{n+1} \sum_{i=1}^{\infty} b_{n+1, i} W_{n, i} u_{n} \| \\
& +\left\|V_{n+1} \sum_{i=1}^{\infty} b_{n+1, i} W_{n, i} u_{n}-V_{n} \sum_{i=1}^{\infty} b_{n, i} W_{n, i} u_{n}\right\| \\
& \leq\left[1-(1-k) \zeta_{n+1}\right] \sum_{i=1}^{\infty} b_{n+1, i}\left\|W_{n+1, i} u_{n+1}-W_{n, i} u_{n}\right\| \\
& +\left\|V_{n+1} \sum_{i=1}^{\infty} b_{n+1, i} W_{n, i} u_{n}-V_{n+1} \sum_{i=1}^{\infty} b_{n, i} W_{n, i} u_{n}\right\|+\left\|\left(V_{n+1}-V_{n}\right) \sum_{i=1}^{\infty} b_{n, i} W_{n, i} u_{n}\right\| \\
& \leq\left[1-(1-k) \zeta_{n+1}\right] \sum_{i=1}^{\infty} b_{n+1, i}\left[\left\|u_{n+1}-u_{n}\right\|+\frac{2 M^{\prime \prime \prime}}{\varepsilon}\left|r_{n+1}-r_{n}\right|+2 M^{\prime \prime \prime}\left|\alpha_{n+1, i}-\alpha_{n, i}\right|\right] \\
& +\| \zeta_{n+1} f\left(\sum_{i=1}^{\infty} b_{n+1, i} W_{n, i} u_{n}\right)+\left(1-\zeta_{n+1}\right) \sum_{i=1}^{\infty} b_{n+1, i} W_{n, i} u_{n} \\
& -\zeta_{n+1} f\left(\sum_{i=1}^{\infty} b_{n, i} W_{n, i} u_{n}\right)-\left(1-\zeta_{n+1}\right) \sum_{i=1}^{\infty} b_{n, i} W_{n, i} u_{n} \| \\
& +\left|\zeta_{n+1}-\zeta_{n}\right|\left\|f\left(\sum_{i=1}^{\infty} b_{n, i} W_{n, i} u_{n}\right)\right\|+\left|\zeta_{n+1}-\zeta_{n}\right|\left\|\sum_{i=1}^{\infty} b_{n, i} W_{n, i} u_{n}\right\| \\
& \leq\left[1-(1-k) \zeta_{n+1}\right]\left\|x_{n+1}-x_{n}\right\|+2 M^{\prime \prime \prime} \sum_{i=1}^{\infty}\left|\alpha_{n+1, i}-\alpha_{n, i}\right|+\frac{6 M^{\prime \prime \prime}}{\varepsilon}\left|r_{n+1}-r_{n}\right| \\
& +4 M^{\prime \prime \prime}\left|\delta_{n+1}-\delta_{n}\right|+2 M^{\prime \prime \prime}\left|\zeta_{n+1}-\zeta_{n}\right|+M^{\prime \prime \prime} \sum_{i=1}^{\infty}\left|b_{n+1, i}-b_{n, i}\right|
\end{aligned}
$$

Then Lemma 1.4 implies that $\lim _{n \rightarrow \infty}\left\|x_{n+1}-x_{n}\right\|=0$.

Since $\zeta_{n} \rightarrow 0$, we have $x_{n+1}-\sum_{i=1}^{\infty} b_{n, i} W_{n, i} u_{n} \rightarrow 0$, as $n \rightarrow \infty$. Since $\delta_{n} \rightarrow 0$, we have $x_{n}-u_{n} \rightarrow 0$, which implies that $\sum_{i=1}^{\infty} b_{n, i} W_{n, i} u_{n}-u_{n} \rightarrow 0$, as $n \rightarrow \infty$.

Step 4. $\lim \sup _{n \rightarrow+\infty}\left\langle f\left(p_{0}\right)-p_{0}, J\left(x_{n+1}-p_{0}\right)\right\rangle \leq 0$.

Noticing Lemmas 1.7 and 2.3, we know that there exists $z_{t}$ such that $z_{t}=\operatorname{tf}\left(z_{t}\right)+(1-$ t) $\sum_{i=1}^{\infty} b_{n, i} W_{n, i} z_{t}$ for $t \in(0,1)$. Moreover, $z_{t} \rightarrow p_{0} \in \Omega$, as $t \rightarrow 0$. $p_{0}$ is the unique solution of the variational inequality (2.14).

Since $\left\|z_{t}\right\| \leq\left\|z_{t}-p_{0}\right\|+\left\|p_{0}\right\|,\left\{z_{t}\right\}$ is bounded, as $t \rightarrow 0$. Using Lemma 1.3, we have

$$
\begin{aligned}
&\left\|z_{t}-u_{n}\right\|^{2} \\
&=\left\|z_{t}-\sum_{i=1}^{\infty} b_{n, i} W_{n, i} u_{n}+\sum_{i=1}^{\infty} b_{n, i} W_{n, i} u_{n}-u_{n}\right\|^{2}
\end{aligned}
$$


Wei et al. Journal of Inequalities and Applications (2016) 2016:22

Page 15 of 20

$$
\begin{aligned}
\leq & \left\|z_{t}-\sum_{i=1}^{\infty} b_{n, i} W_{n, i} u_{n}\right\|^{2}+2\left\langle\sum_{i=1}^{\infty} b_{n, i} W_{n, i} u_{n}-u_{n}, J\left(z_{t}-u_{n}\right)\right\rangle \\
= & \left\|t f\left(z_{t}\right)+(1-t) \sum_{i=1}^{\infty} b_{n, i} W_{n, i} z_{t}-\sum_{i=1}^{\infty} b_{n, i} W_{n, i} u_{n}\right\|^{2} \\
& +2\left\langle\sum_{i=1}^{\infty} b_{n, i} W_{n, i} u_{n}-u_{n}, J\left(z_{t}-u_{n}\right)\right\rangle \\
\leq & \left\|z_{t}-u_{n}\right\|^{2}+2 t\left\langle f\left(z_{t}\right)-\sum_{i=1}^{\infty} b_{n, i} W_{n, i} z_{t}, J\left(z_{t}-\sum_{i=1}^{\infty} b_{n, i} W_{n, i} u_{n}\right)\right\rangle \\
& +2\left\langle\sum_{i=1}^{\infty} b_{n, i} W_{n, i} u_{n}-u_{n}, J\left(z_{t}-u_{n}\right)\right\rangle \\
\leq & \left\|z_{t}-u_{n}\right\|^{2}+2 t\left\langle f\left(z_{t}\right)-\sum_{i=1}^{\infty} b_{n, i} W_{n, i} z_{t}, J\left(z_{t}-\sum_{i=1}^{\infty} b_{n, i} W_{n, i} u_{n}\right)\right\rangle \\
& +2\left\|\sum_{i=1}^{\infty} b_{n, i} W_{n, i} u_{n}-u_{n}\right\|\left\|z_{t}-u_{n}\right\|,
\end{aligned}
$$

which implies that

$$
t\left\langle\sum_{i=1}^{\infty} b_{n, i} W_{n, i} z_{t}-f\left(z_{t}\right), J\left(z_{t}-\sum_{i=1}^{\infty} b_{n, i} W_{n, i} u_{n}\right)\right\rangle \leq\left\|\sum_{i=1}^{\infty} b_{n, i} W_{n, i} u_{n}-u_{n}\right\|\left\|z_{t}-u_{n}\right\| .
$$

So, $\lim _{t \rightarrow 0} \lim \sup _{n \rightarrow+\infty}\left\langle\sum_{i=1}^{\infty} b_{n, i} W_{n, i} z_{t}-f\left(z_{t}\right), J\left(z_{t}-\sum_{i=1}^{\infty} b_{n, i} W_{n, i} u_{n}\right)\right\rangle \leq 0$ in view of Step 3.

Since $z_{t} \rightarrow p_{0}$, we have $\sum_{i=1}^{\infty} b_{n, i} W_{n, i} z_{t} \rightarrow \sum_{i=1}^{\infty} b_{n, i} W_{n, i} p_{0}=p_{0}$, as $t \rightarrow 0$. Noticing the fact that

$$
\begin{aligned}
\left\langle p_{0}-\right. & \left.f\left(p_{0}\right), J\left(p_{0}-\sum_{i=1}^{\infty} b_{n, i} W_{n, i} u_{n}\right)\right\rangle \\
= & \left\langle p_{0}-f\left(p_{0}\right), J\left(p_{0}-\sum_{i=1}^{\infty} b_{n, i} W_{n, i} u_{n}\right)-J\left(z_{t}-\sum_{i=1}^{\infty} b_{n, i} W_{n, i} u_{n}\right)\right\rangle \\
& +\left\langle p_{0}-f\left(p_{0}\right), J\left(z_{t}-\sum_{i=1}^{\infty} b_{n, i} W_{n, i} u_{n}\right)\right\rangle \\
= & \left\langle p_{0}-f\left(p_{0}\right), J\left(p_{0}-\sum_{i=1}^{\infty} b_{n, i} W_{n, i} u_{n}\right)-J\left(z_{t}-\sum_{i=1}^{\infty} b_{n, i} W_{n, i} u_{n}\right)\right\rangle \\
& +\left\langle p_{0}-f\left(p_{0}\right)-\sum_{i=1}^{\infty} b_{n, i} W_{n, i} z_{t}+f\left(z_{t}\right), J\left(z_{t}-\sum_{i=1}^{\infty} b_{n, i} W_{n, i} u_{n}\right)\right\rangle \\
& +\left\langle\sum_{i=1}^{\infty} b_{n, i} W_{n, i} z_{t}-f\left(z_{t}\right), J\left(z_{t}-\sum_{i=1}^{\infty} b_{n, i} W_{n, i} u_{n}\right)\right\rangle
\end{aligned}
$$

we have $\lim \sup _{n \rightarrow+\infty}\left\langle p_{0}-f\left(p_{0}\right), J\left(p_{0}-\sum_{i=1}^{\infty} b_{n, i} W_{n, i} u_{n}\right)\right\rangle \leq 0$. 
Since $\left\langle p_{0}-f\left(p_{0}\right), J\left(p_{0}-x_{n+1}\right)\right\rangle=\left\langle p_{0}-f\left(p_{0}\right), J\left(p_{0}-x_{n+1}\right)-J\left(p_{0}-\sum_{i=1}^{\infty} b_{n, i} W_{n, i} u_{n}\right)\right\rangle+\left\langle p_{0}-\right.$ $\left.f\left(p_{0}\right), J\left(p_{0}-\sum_{i=1}^{\infty} b_{n, i} W_{n, i} u_{n}\right)\right\rangle$ and $x_{n+1}-\sum_{i=1}^{\infty} b_{n, i} W_{n, i} u_{n} \rightarrow 0$ in view of Step 3 , we have $\lim \sup _{n \rightarrow \infty}\left\langle f\left(p_{0}\right)-p_{0}, J\left(x_{n+1}-p_{0}\right)\right\rangle \leq 0$.

Step 5. $x_{n} \rightarrow p_{0}$, as $n \rightarrow+\infty$, where $p_{0} \in \Omega$ is the same as in Step 4 .

Since

$$
\left\|u_{n}-p_{0}\right\| \leq\left(1-\delta_{n}\right)\left\|x_{n}-p_{0}\right\|+\delta_{n}\left\|\frac{x_{n}+u_{n}}{2}-p_{0}\right\|
$$

we have

$$
\left\|u_{n}-p_{0}\right\| \leq\left\|x_{n}-p_{0}\right\|
$$

Using Lemma 1.3, we have, for $n \geq 0$,

$$
\begin{aligned}
& \left\|x_{n+1}-p_{0}\right\|^{2} \\
& =\left\|V_{n} \sum_{i=1}^{\infty} b_{n, i} W_{n, i} u_{n}-p_{0}\right\|^{2} \\
& =\left\|V_{n} \sum_{i=1}^{\infty} b_{n, i} W_{n, i} u_{n}-V_{n} \sum_{i=1}^{\infty} b_{n, i} W_{n, i} p_{0}+V_{n} p_{0}-p_{0}\right\|^{2} \\
& \leq\left[1-(1-k) \zeta_{n}\right]\left\|u_{n}-p_{0}\right\|^{2}+2\left\langle V_{n} p_{0}-p_{0}, J\left(x_{n+1}-p_{0}\right)\right\rangle \\
& \leq\left[1-(1-k) \zeta_{n}\right]\left\|x_{n}-p_{0}\right\|^{2}+2 \zeta_{n}\left\langle f\left(p_{0}\right)-p_{0}, J\left(x_{n+1}-p_{0}\right)\right\rangle .
\end{aligned}
$$

Using Lemma 1.4, the assumptions and the result of Step 4, we know that $x_{n} \rightarrow p_{0}$, as $n \rightarrow+\infty$. Combining with the result of Step $3, u_{n} \rightarrow p_{0}$, as $n \rightarrow \infty$.

Copy Step 5 in Theorem 2.2, $z_{n} \rightarrow p_{0}$, as $n \rightarrow \infty$.

This completes the proof.

Remark 2.8 The assumptions imposed on the real number sequences in Theorem 2.7 are reasonable if we take $\alpha_{n, i}=\frac{1}{(n+1) i^{2}}, \delta_{n}=\zeta_{n}=\frac{1}{n+1}, b_{n, i}=\frac{n+1}{(n+2)^{i}}$, and $r_{n}=\varepsilon+\frac{1}{n+1}$, for $n \geq 0$ and $i=1,2, \ldots$.

Remark 2.9 The four sequences $\left\{x_{n}\right\},\left\{u_{n}\right\},\left\{y_{n}\right\}$, and $\left\{z_{n}\right\}$ in Theorem 2.2 and the three sequences $\left\{u_{n}\right\},\left\{x_{n}\right\}$, and $\left\{z_{n}\right\}$ in Theorem 2.7 are proved to be strongly convergent to the zero point of an $m$-accretive mapping and the fixed point of an infinite family of nonexpansive mappings. The strongly convergent point is proved to be the unique solution of one kind variational inequalities.

Remark 2.10 In Theorem 2.7, $V_{n}$ can be regarded as an averaged mapping, whose definition can be seen in [16].

Remark 2.11 The discussions on Theorems 2.2 and 2.7 are undertaken in the frame of a real smooth and uniformly convex Banach space, which is more general than that in Hilbert space. 


\section{Examples and numerical experiments}

In this section, we provide some numerical experiments to show that both algorithms (A) and (B) are effective. In our experiments, we consider the following examples.

Example 3.1 In algorithm (A), suppose $E=C=(-\infty,+\infty)$. Let $a_{i}=1, \alpha_{n, i}=\frac{1}{2^{n+i}}, b_{n, i}=$ $\frac{n+1}{(n+2)^{i}}, \gamma_{n}=\delta_{n}=\beta_{n}=\frac{1}{n+1}, r_{n}=\frac{n+2}{n+1}, \varepsilon=1, f(x)=\frac{x}{14}, k=\frac{1}{7}, A x=\frac{x}{2}, T x=\frac{2 x}{7}, \bar{\gamma}=\frac{2}{7}, \eta=\frac{1}{2}$, and $S_{i} x=\frac{x}{2^{i}}$. Then all of the assumptions in Theorem 2.2 are satisfied. And $\Omega=\bigcap_{i=1}^{\infty} \operatorname{Fix}\left(S_{i}\right) \cap$ $A^{-1} 0=\{0\}$.

Table 1 The values of $\left\{y_{n}\right\},\left\{u_{n}\right\},\left\{x_{n}\right\}$, and $\left\{z_{n}\right\}$ with initial value $x_{0}=-4$

\begin{tabular}{|c|c|c|c|c|}
\hline$n$ & $y_{n}$ & $u_{n}$ & $x_{n}$ & $z_{n}$ \\
\hline 0 & -4.0000000000 & 1.333333333333333 & -4.0000000000000 & \\
\hline 1 & -0.8477427 & 635807052254677 & .0952380952381 & -1.0952380952381 \\
\hline 2 & -0.408616 & & & \\
\hline 3 & & & & \\
\hline 4 & -0.1335324 & -0.122849839925766 & -0.191673798999402 & -0.543274417197196 \\
\hline 5 & -0.08100538 & -0.075755030882579 & -0.117198947637147 & -0.458059323285186 \\
\hline 6 & -0.05007082 & -0.047345876009489 & .072845261243154 & -0.393856979611514 \\
\hline 7 & & & & \\
\hline 8 & -0.01 & & .029008549436138 & -0.304741934734471 \\
\hline 9 & & & & \\
\hline 10 & & & & 5709 \\
\hline 11 & & & & \\
\hline 12 & & & & \\
\hline 13 & -0.002 & & & \\
\hline 14 & -0.001388 & & & \\
\hline 15 & & & & \\
\hline 16 & & & & \\
\hline 17 & & & & -0.1 \\
\hline 18 & & & & \\
\hline 19 & & & & \\
\hline 20 & & & & -0.1 \\
\hline 21 & & & & 80834 \\
\hline 22 & -0.0000452522 & -0.00004 & -0.00 & 50767 \\
\hline 23 & -0.0000296392 & -0.0000292103517604 & -0.000044118782606 & 620117369 \\
\hline 24 & -0.0000194276 & -0.000019158216414 & -0.000028928262428 & -0.10374138295674 \\
\hline 25 & & -0.000012573398811 & -0.000018980591458 & -0.099592486862135 \\
\hline 26 & & & & \\
\hline 27 & & & & \\
\hline 28 & -0.0000036092 & -0.000003566 & -0.000005380130737 & 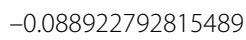 \\
\hline 29 & & & -0.000003537814776 & -0.085856611608568 \\
\hline 30 & -0.0000015607 & -0.000001543347773 & -0.000002327427839 & -0.08299480213587 \\
\hline 31 & 0.0000000000 & 0.000000000000000 & -0.000001531804738 & -0.08031759986713 \\
\hline
\end{tabular}

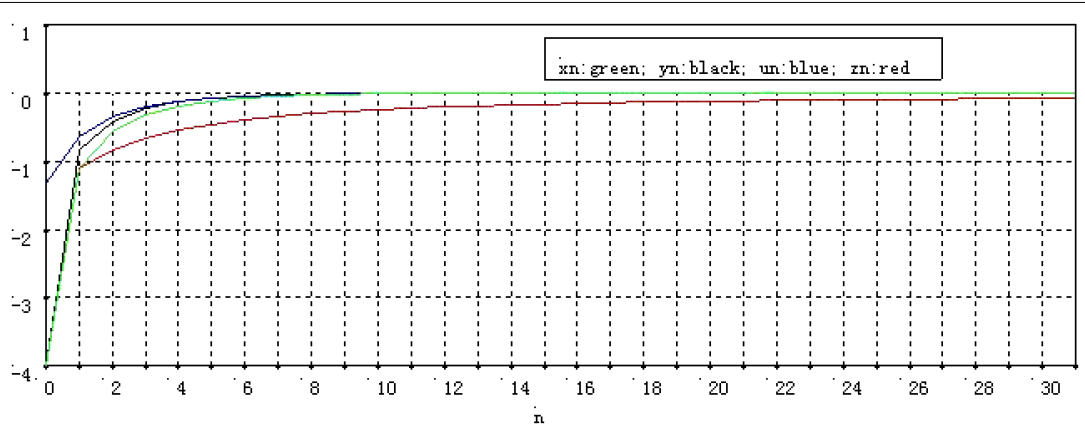

Figure 1 The convergence of $\left\{y_{n}\right\},\left\{u_{n}\right\},\left\{x_{n}\right\}$, and $\left\{z_{n}\right\}$. 

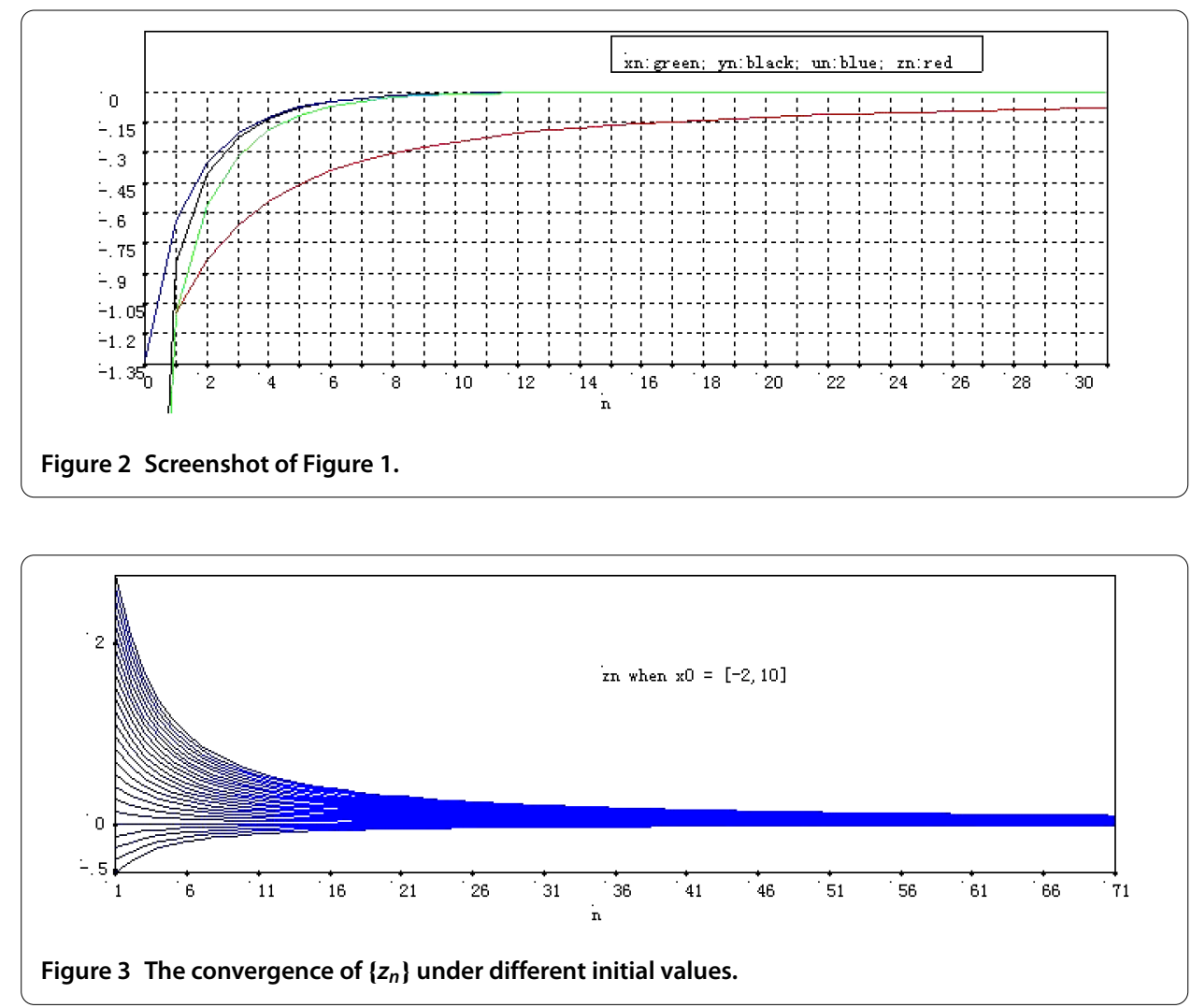

Table 2 The values of $\left\{u_{n}\right\},\left\{x_{n}\right\}$, and $\left\{z_{n}\right\}$ with initial value $x_{0}=8$

\begin{tabular}{rlll}
\hline $\boldsymbol{n}$ & $\boldsymbol{x}_{\boldsymbol{n}}$ & $\boldsymbol{u}_{\boldsymbol{n}}$ & $\boldsymbol{z}_{\boldsymbol{n}}$ \\
\hline 0 & 8.0000000000000 & 2.6666666666667 & \\
1 & 0.0907029478458 & 0.0680272108844 & 0.09070295 \\
2 & 0.0199727386316 & 0.0170138143899 & 0.05533785 \\
3 & 0.0068807569080 & 0.0061640113968 & 0.03918548 \\
4 & 0.0028754047605 & 0.0026453723797 & 0.03010796 \\
5 & 0.0013373257810 & 0.0012506472582 & 0.02435383 \\
6 & 0.0006653502921 & 0.0006291407524 & 0.02040576 \\
7 & 0.0003466462534 & 0.0003303972103 & 0.01754017 \\
8 & 0.0001867447646 & 0.0001790597949 & 0.01537099 \\
9 & 0.0001031871958 & 0.0000994036653 & 0.01367457 \\
10 & 0.0000581631893 & 0.0000562404392 & 0.01231293 \\
11 & 0.0000333151231 & 0.0000323125847 & 0.01119660 \\
12 & 0.0000193365400 & 0.0000188025922 & 0.01026516 \\
13 & 0.0000113483809 & 0.0000110588814 & 0.00947641 \\
14 & 0.0000067234088 & 0.0000065640391 & 0.00879999 \\
15 & 0.0000040158582 & 0.0000039269655 & 0.00821360 \\
16 & 0.0000024157047 & 0.0000023655516 & 0.00770040 \\
17 & 0.0000014622202 & 0.0000014336377 & 0.00724752 \\
18 & 0.0000008899717 & 0.0000008735364 & 0.00684493 \\
19 & 0.0000005443463 & 0.0000005348202 & 0.00648470 \\
20 & 0.0000003344191 & 0.0000003288581 & 0.00616048 \\
\hline
\end{tabular}

Remark 3.1 All codes were written in Visual Basic Six. For the initial value $x_{0}=-4$, the values of $\left\{y_{n}\right\},\left\{u_{n}\right\},\left\{x_{n}\right\}$, and $\left\{z_{n}\right\}$ with different $n$ are reported in Table 1 .

Remark 3.2 In Figure 1, the abscissa denotes the iterative step and the ordinate denotes the values of $\left\{y_{n}\right\},\left\{u_{n}\right\},\left\{x_{n}\right\}$, and $\left\{z_{n}\right\}$ with different iterative step $n$. 

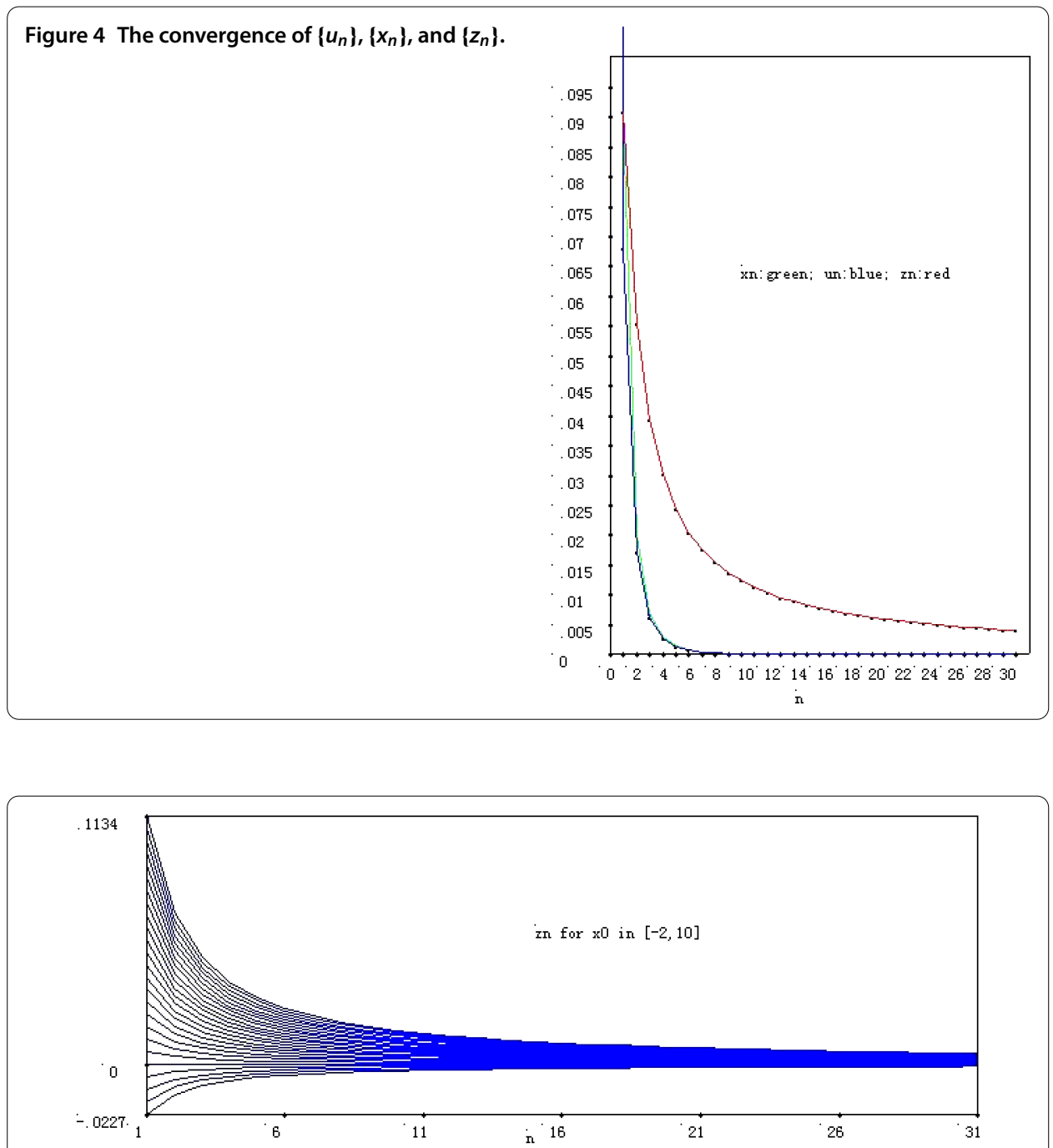

Figure 5 The convergence of $\left\{z_{n}\right\}$ for different initial values.

Remark 3.3 Table 1 and Figure 1 show that the sequences $\left\{y_{n}\right\},\left\{u_{n}\right\},\left\{x_{n}\right\}$, and $\left\{z_{n}\right\}$ converge to 0 . Also, $\{0\}=\Omega$.

Remark 3.4 Figure 2 is a screen shot of Figure 1, whose ordinates are enlarged. Our purpose to draw Figure 2 is to let Figure 1 be clearer.

Remark 3.5 Figure 3 shows the values of ergodic sequence $\left\{z_{n}\right\}$ with the initial value $x_{0}$ being chosen arbitrarily in $[-2,10]$.

Example 3.2 In algorithm (B), suppose $E=(-\infty,+\infty)$ and $C=[-2,10]$. Let $a_{i}=1, \alpha_{n, i}=$ $\frac{1}{2^{n+i}}, b_{n, i}=\frac{n+1}{(n+2)^{i}}, \delta_{n}=\zeta_{n}=\frac{1}{n+1}, r_{n}=\frac{n+2}{n+1}, \varepsilon=1, f(x)=\frac{x}{14}, k=\frac{1}{7}, A x=\frac{x}{2}$, and $S_{i} x=\frac{x}{2^{i}}$. Then all of the assumptions in Theorem 2.7 are satisfied. Also $\Omega=\bigcap_{i=1}^{\infty} \operatorname{Fix}\left(S_{i}\right) \cap A^{-1} 0=\{0\}$.

All codes were written in Visual Basic Six, the values of $\left\{u_{n}\right\},\left\{x_{n}\right\}$, and $\left\{z_{n}\right\}$ with different $n$ are reported in Table 2 . 
Remark 3.6 Table 2 and Figure 4 show that the sequences $\left\{u_{n}\right\},\left\{x_{n}\right\}$, and $\left\{z_{n}\right\}$ converge to 0 . Also, $\{0\}=\Omega$.

Remark 3.7 Figure 5 shows the values of ergodic sequence $\left\{z_{n}\right\}$ with the initial value $x_{0}$ being chosen arbitrarily in $[-2,10]$.

Competing interests

The authors declare that they have no competing interests.

\section{Authors' contributions}

The first and the third authors are responsible for the first and the second sections. The second author is responsible for the experiment in Section 3. All authors read and approve the final manuscript.

\section{Author details}

${ }^{1}$ School of Mathematics and Statistics, Hebei University of Economics and Business, Shijiazhuang, 050061, China. ${ }^{2}$ College of Humanities and Development Studies, China Agricultural University, Beijing, 100083, China. ${ }^{3}$ Department of Mathematics, Texas A\&M University-Kingsville, Kingsville, TX 78363, USA. ${ }^{4}$ Department of Mathematics, Faculty of Science, King Abdulaziz University, Jeddah, 21589, Saudi Arabia.

\section{Acknowledgements}

This research was supported by the National Natural Science Foundation of China (11071053), the Natural Science Foundation of Hebei Province (A2014207010), the Key Project of Science and Research of Hebei Educational Department (ZH2012080) and the Key Project of Science and Research of Hebei University of Economics and Business (2015KYZ03).

Received: 15 October 2015 Accepted: 7 January 2016 Published online: 21 January 2016

\section{References}

1. Cai, G, Bu, S: Approximation of common fixed points of a countable family of continuous pseudocontractions in a uniformly smooth Banach space. Appl. Math. Lett. 24(2), 1998-2004 (2001)

2. Agarwal, RP, O'Regan, D, Sahu, DR: Fixed Point Theory for Lipschitz-Type Mappings with Applications. Springer, Berlin (2008)

3. Takahashi, W: Proximal point algorithms and four resolvents of nonlinear operators of monotone type in Banach spaces. Taiwan. J. Math. 12(8), 1883-1910 (2008)

4. Baillon, J-B: Un théorème de type ergodique pour les contractions non linéaires dans un espace de Hilbert. C. R. Acad. Sci. Paris Sér. A-B 280(22), Aii, A1511-A1514 (1975) (in French)

5. Alghamdi, MA, Alghamdi, MA, Shahzad, N, Xu, HK: The implicit midpoint rule for nonexpansive mappings. Fixed Point Theory Appl. 2014, 96 (2014)

6. Xu, HK, Alghamdi, MA, Shahzad, N: Ergodicity of the implicit midpoint rule for nonexpansive mappings. J. Inequal. Appl. 2015, 4 (2015)

7. Marino, G, Xu, HK: A general iterative method for nonexpansive mappings in Hilbert spaces. J. Math. Anal. Appl. 318, 43-52 (2006)

8. Takahashi, W: Nonlinear Functional Analysis. Fixed Point Theory and Its Application. Yokohama Publishers, Yokohama (2000)

9. Browder, FE: Semicontractive and semiaccretive mappings in Banach spaces. Bull. Am. Math. Soc. 74, 660-665 (1968)

10. Ceng, LC, Khan, AR, Ansari, QH, Yao, JC: Strong convergence of composite iterative schemes for zeros of $m$-accretive operators in Banach spaces. Nonlinear Anal. 70, 1830-1840 (2009)

11. Liu, LS: Ishikawa and Mann iterative process with errors for nonlinear strongly accretive mappings in Banach spaces J. Anal. Appl. 194, 114-125 (1995)

12. Barbu, V: Nonlinear Semigroups and Differential Equations in Banach Space. Noordhoff, Leyden (1976)

13. Cai, G, Hu, CS: Strong convergence theorems of a general iterative process for a finite family of $\lambda_{j}$-strictly pseudo-contractions in q-uniformly smooth Banach space. Comput. Math. Appl. 59, 149-160 (2010)

14. Wei, L, Duan, LL: A new iterative algorithm for the sum of two different types of finitely many accretive operators in Banach space and its connection with capillarity equation. Fixed Point Theory Appl. 2015, 25 (2015)

15. Bruck, RE: Properties of fixed-point sets of nonexpansive mappings in Banach spaces. Trans. Am. Math. Soc. 179 251-262 (1973)

16. Yao, Y: A general iterative method for a finite family of nonexpansive mappings. Nonlinear Anal. 66, 2676-2678 (2007) 